\title{
Investigando enfermedades raras del adulto en atención primaria: una hipótesis clasificatoria
}

\author{
M. ORTEGA CALVO, F. GARCÍA DE LA CORTE', P. IGLESIAS BONILLA² \\ Centro de Salud Esperanza Macarena. ${ }^{I}$ Centro de Salud San Pablo. ${ }^{2}$ Distrito Sanitario. Sevilla
}

RARE DISEASES AT A PRIMARY CARE FACILITY

\begin{abstract}
RESUMEN
Las enfermedades raras (ER) han de ser un objetivo de investigación en atención primaria por su potencial de productividad científico-biológica. Nos parece necesaria una sistematización de los resultados. Hemos realizado este trabajo retrospectivo longitudinal que recoge los hallazgos más importantes de nuestro interés en las ER de base genética. Los resultados se han clasificado en: a) observación y comunicación de reacciones adversas a medicamentos raras (RAMR); b) diagnóstico de entidades nosológicas raras (ENR); y c) estudio de prevalencia de ER (PER).
\end{abstract}

PALABRAS CLAVE: Enfermedad del suero "like". Cefonicida. Hiperprolactinemia. Verapamilo. Enfermedad granulomatosa crónica. Déficit en p67-phox. Hemorragia subaracnoidea aguda. Déficit en el "pool" de plaquetas. Distrofia miotónica tipo I. Racimo.

\begin{abstract}
A pragmatic classification. Rare diseases $(R D)$ might be a research target on primary care because their gift of scientific knowledge building. A rational scheme would be necessary for clinical and scientific findings.Retrospective long-term report of the most important $R D$ achieved for a ten years period by a general practitioner at a non-urban primary care facility (Andalusia-Spain). Our results are classified as: a) rare adverse drug reactions $(R A D R) ; b)$ accurate $R D$ diagnosis $(R D D)$; and c) $R D$ prevalence study $(R D P)$.
\end{abstract}

KEY WORDS: Serum sickness "like”. Cefonicid. Hyperprolactinemia. Verapamil. Chronic granulomatous disease. P67-phox deficiency. Acute subarachnoid hemorrhage. Platelet storage pool disease. Type I myotonic dystrophy. Cluster.

Ortega Calvo M, García de la Corte F, Iglesias Bonilla. Investigando enfermedades raras del adulto en atención primaria: una hipótesis clasificatoria. An Med Interna (Madrid) 2007; 24: 535-538.

\section{INTRODUCCIÓN}

Las enfermedades raras (ER) deben de constituir un objetivo de investigación en atención primaria porque son muy productivas desde un punto de vista biológico si se estudian y analizan con rigor (1-2). Los avances en el diagnóstico molecular y la flexibilidad que nos permite Internet hacen accesibles las ER a los médicos de familia en los centros de salud (2). En este artículo hemos querido responder a la pregunta siguiente: ¿Cómo podemos clasificar los hallazgos en ER desde atención primaria de tal modo que la información sea lo más rentable posible a la comunidad científica?

\section{MATERIAL Y MÉTODOS}

Para intentar responder hemos realizado un análisis longitudinal y retrospectivo de unos 10 años aproximadamente (19922002). El entorno ha sido una consulta de medicina general atendida de forma continua por un especialista en medicina de familia con interés en las ER. La consulta estaba ubicada en un centro de salud de la provincia de Sevilla (Zona Básica de Pilas-Sevilla-España): Hemos clasificado los resultados en tres apartados que ayudan a organizar los contenidos racionalmente: a) observación y comunicación de reacciones adversas a medicamentos raras (RAMR); b) diagnóstico de entidades nosológicas raras (ENR); y c) estudio de prevalencia de ER.

Estos apartados se refieren primordialmente a ER con base genética. La forma de intercambiar la información con los especialistas hospitalarios y con los expertos ha sido mediante el contacto directo, con desplazamientos al centro hospitalario o al laboratorio de referencia. También por medio del teléfono, del servicio nacional de correos y finalmente por medio del correo electrónico que es un instrumento de especial utilidad para la investigación y el manejo de las ER en atención primaria (2-5). La sospecha de los casos de RAMR fue iniciada mediante la comunicación de la tarjeta amarilla a la Unidad de Farmacovigilancia Regional.

Trabajo aceptado: 4 de octubre de 2007

Correspondencia: Manuel Ortega Calvo. Avda. de la Cruz del Campo, 36. Bloque 1,2A. 41005 Sevilla. e-mail: ortegacalvo@terra.es 


\section{RESULTADOS}

- RAMR. La primera fué un cuadro "parecido" a la enfermedad del suero (6) que observamos en un paciente varón de 44 años que había sido tratado con cefonicida IM por una complicación post-quirúrgica. Hubo una total recuperación de la artritis y de la proteinuria y no han existido repeticiones del episodio en un período posterior de observación de ocho años. La segunda fue una hiperprolactinemia (documentada desde primaria y no derivada a ningún otro nivel) causada por el tratamiento con verapamilo en una mujer hipertensa de 37 años de edad (7). Después de la retirada del fármaco no se ha vuelto a repetir el evento tras una observación de siete años con controles de prolactina seriados.

El tercer caso ha sido quizás el más demostrativo para nosotros. Se trataba de una mujer de 65 años que estaba en tratamiento con 20 mgrs. de enalapril cada 24 horas por hipertensión arterial. Tuvo varios episodios de dolor abdominal epigástrico y peri umbilical, documentado en dos ocasiones en las urgencias hospitalarias con elevación de la amilasa pancreática plasmática y de la amilasuria a unos niveles que conformaban el diagnóstico de pancreatitis. Los estudios de imagen (eco y TAC) y la colangio-pancreatografía-retrógrada endoscópica (CPRE) fueron negativas en un ingreso por este cuadro. El Programa de Detección de Reacciones Adversas a Medicamentos de la Unidad de Farmacovigilancia del hospital detectó a esta paciente como posible portadora de una pancreatitis por enalaprilo. Se le retiró el fármaco y se le instauró tratamiento con un bloqueante de los canales lentos del calcio, siendo observada muy de cerca desde entonces. Después de tres años de evolución sin dolor abdominal nos decidimos a comunicarla en un congreso nacional. Tras siete años de observación sin tratamiento con enalaprilo y cuando teníamos preparado el manuscrito para publicarla en una revista especializada hizo un nuevo cuadro de dolor abdominal con elevación de amilasa, estando esta vez en tratamiento con un bloqueante de los canales lentos del calcio. Se había roto el criterio de causalidad (8-9).

-ENR. El primero de ellos fue una mujer de 38 años, fumadora de 30 cigarrillos/24 horas que hizo una lesión inflamatoria en el hemitórax derecho. Tras seis meses de estudio en consultas externas de Neumología, un ingreso hospitalario y una toracotomía exploradora se llegó a la conclusión de que padecía una granulomatosis broncocéntrica con aspergilosis no invasiva y un déficit en la función oxidativa de los polimorfonucleares. Pudimos contactar con A. W. Segal (10-11), investigador de la Universidad de Londres. Le expusimos el caso y él se brindó a realizar el diagnóstico bioquímico. A los pocos meses recibimos el informe de que esta paciente padecía una enfermedad granulomatosa crónica por déficit en p-67 phox (12). Diez años después, tiene una calidad de vida aceptable aunque ha tenido que sufrir un transplante de córnea por las secuelas de una conjuntivitis bacteriana.

La otra ENR ocurrió también en una mujer, esta vez de 45 años, sin antecedentes de hipertensión arterial que sufrió bruscamente una caída al suelo con pérdida conciencia mientras estaba sentada en un bar de la localidad. Fue trasladada al servicio de urgencias en donde se le objetivó rigidez de nuca, sin fiebre y una situación de coma neurológico, evacuándose rápidamente al hospital. Allí se le diagnosticó de una hemorragia subaracnoidea (HSA) sin causa evidente, pues se le habían realizado dos angiografías que habían resultado nor- males y no se le había detectado ninguna alteración médica. La enferma regresó a su casa con el diagnóstico de HSA idiopática para seguimiento en su centro de salud. Tuvimos la suerte de que en nuestra historia clínica había registrado un episodio de hemorragia ocular externa que había sido diagnosticado por un oftalmólogo como una episcleritis aguda dos años antes de la HSA. Asumimos la hipótesis de que aquella hemorragia en la cámara anterior ocular y la ocurrida en el espacio subaracnoideo podrían tener la misma causa y derivamos a la paciente para intentar despistar un trastorno de coagulación. Tras dos años de pruebas biológicas en una unidad especializada de nivel terciario (13-14), se le diagnosticó de un déficit en el pool de plaquetas (15) asociado a la talasemia minor que anteriormente ya se sabía que padecía esta mujer.

- Prevalencia de ER. Desde que nos incorporamos al centro empezamos a detectar una elevada morbididad de un tipo de distrofia muscular congénita, la enfermedad de Steinert. Tras años de observación, decidimos realizar un estudio de prevalencia. Llevamos a cabo una recopilación (case-finding) de estos pacientes (16) llegando a la conclusión mediante un sencillo análisis (proporciones de prevalencia basadas en hipótesis muestrales) de que en nuestra zona la enfermedad de Steinert no es una ER porque existe un "racimo". Hay otros lugares del Estado español en donde esto también ocurre $(17,18)$.

\section{DISCUSIÓN}

Las características intrínsecas de la atención primaria rural en nuestro país (19-20) la hacen esencialmente diferente de la atención primaria urbana. El esfuerzo investigador es por lo tanto más difícil y exige una alta motivación por parte del profesional. Vistos los resultados creemos que el interés en las ER desde un centro de salud es eficiente desde un punto de vista biológico. La sistematización de nuestro trabajo en los apartados de fármaco vigilancia de RAMR, diagnóstico de ENR y análisis de la prevalencia de ER es una hipótesis clasificatoria de resultados en este campo de investigación para atención primaria. Es la aportación metodológica más relevante de este artículo y una forma de responder a nuestra pregunta.

Es evidente que el nivel primario constituye un lugar estratégico para la observación de las Reacciones Adversas a Medicamentos y más aún si son raras (RAMR) (21-23). Las dos que hemos elegido para este artículo (enfermedad del suero "like" tras la utilización de cefonicida IM e hiperprolactinemia por verapamil) (6-7) creemos que ilustran tal afirmación. Pero la experiencia de la tercera, la pseudo pancreatitis por enalapril ha sido sin duda la más enriquecedora para nosotros, haciéndonos pensar en la necesidad de unos criterios de causalidad específicos para nuestro nivel (21-23) o en la necesidad de que se refrenden los criterios convencionales longitudinalmente en el tiempo.

El caso de la mujer con déficit en p-67 phox es fruto de una colaboración cercana con el nivel especializado. La alteración bioquímica es una de las formas más raras de EGC (12). Este hallazgo fue informado al Registro Español de Inmunodeficiencias Primarias (REDIP) dirigido por la Dra. Nuria Matamoros en el Hospital Son Dureta de Mallorca (24-26). El nuestro fue el primero notificado desde Andalucía con déficit en p-67 phox. La prevalencia de la enfermedad granulomatosa crónica en todas sus formas (ligadas a cromosoma X y autosómicas recesivas) se estima entre $1 / 200.000$ (27) y $1 / 287.000$ 
nacidos vivos (28). La donación altruista de sangre por parte de esta paciente ha dado substrato material en algún momento a dos líneas de investigación diferentes, la del Profesor Francisco Sobrino en el Departamento de Bioquímica de la Universidad de Sevilla (29-31) y la de la Dra. Berta Sánchez del Servicio de Inmunología de los Hospitales Universitarios Virgen del Rocío también de Sevilla (32). Hemos tenido también la satisfacción de haber generado una observación similar (33).

La HSA queda sin diagnóstico etiológico entre el 10 y el $20 \%$ de los casos según las series (34). La asociación encontrada en nuestro caso (15) (HSA, déficit en el pool de plaquetas y talasemia minor) no está descrita en la bibliografía anteriormente. Tan sólo encontramos un caso de déficit en el pool de plaquetas y HSA (34). Creemos que es una aportación muy interesante para intentar disminuir ese porcentaje de HSAs que queda sin diagnóstico etiológico y además ha tenido consecuencias preventivas para la paciente por el tratamiento profiláctico con desmopresina que ha de recibir ante cualquier maniobra quirúrgica (15).

La distrofia miotónica tipo I o enfermedad de Steinert es una enfermedad rara (16). El interés por cuantificarla en una zona determinada es el intentar desmitificarla de su rareza y el asumir un cierto grado de alerta asistencial ante la aparición de casos no floridos en la misma zona. Estos últimos se pueden expresar sencillamente por un bloqueo aurículo-ventricular de primer grado o por la aparición de algún tipo de opacidad en el cristalino de personas jóvenes. Los valores predictivos de estos dos hallazgos en una zona prevalente (36-38) debieran de ser más elevados que en otras. Sin embargo esta última afirmación es muy discutible dadas las evidencias existentes del valor que posee el diagnóstico genético en las formas paucisintomáticas o atípicas de la distrofia miotónica tipo I $(38,39)$. Según Sciliano et al. (39), cuando se hace un estudio epidemiológico sobre casos floridos queda más de la mitad de formas paucisintomáticas sin registrar.La incertidumbre científica con la que se trabaja en primaria es mayor, a priori, que la existente en el nivel especializado $(2,40)$. Manejar esa incertidumbre de forma apropiada es un objetivo del investigador en ER. La observación longitudinal es una ventaja distintiva que tiene la investigación en primaria sobre la especializada. Un claro ejemplo es la pseudo pancreatitis por enalaprilo $(8,9)$. En este artículo nos hemos ocupado principalmente de RAMR y ER de base genética. Está claro también que el nivel primario es esencial en el diagnóstico e investigación epidemiológica de las enfermedades transmisibles emergentes (41-43).El médico de familia, aún trabajando en el ámbito rural, puede colaborar de forma directa en la evolución de las especialidades básicas si se interesa por las ER $(1,2,40-43)$.

\section{AGRADECIMIENTOS}

Trabajo financiado en parte con la ayuda a la investigación de la Conserjería de Salud de la Junta de Andalucía. Exp. 253/95/85. M.O-C. agradece a la Dra. Isabel Fernández Fernández (Distrito Sanitario Aljarafe y Grupo de Investigación PAI-CTS-382) la confianza y el estímulo que siempre le ha dispensado y también agradece la formación investigadora recibida en la Escuela Andaluza de Salud Pública de Granada (Prof. E. Sánchez-Cantalejo).

\section{Bibliografía}

1. Izquierdo Martínez M, Avellaneda Fernández A. Interdisciplinary approach of rare diseases: A new challenge for a new century. Med Clin (Barc) 2003; 121: 299-303.

2. Ortega Calvo, M. Las enfermedades raras como paradigma científico en atención primaria. Cuadernos de Gestión 2004; 10: 41-4.

3. Temte JL, Anderson AL. Rapid assessment of agents of biological terrorism: Defining the differential diagnosis of inhalational anthrax using electronic communication in a practice-based research network. Ann Fam Med 2004; 2: 434-7.

4. Patterson V, Humphreys J, Chua R. E-mail triage of new neurological outpatient referrals from general practice. J Neurol Neurosurg Psychiatry $2004 ; 75: 617-20$.

5. Lasker JN, Sogolow ED, Sharim RR.: The role of an online community for people with a rare disease: content analysis of messages posted on a primary biliary cirrhosis mailinglist. J Med Internet Res 2005; 7: e10. http://www.jmir.org/2005/1/e10/

6. Ortega Calvo M, Méndez Mora JL, Soriano Crespo E, Fernández Martínez $\mathrm{D}$, Torello Iserte $\mathrm{J}$, et al. Adverse reaction to cefonicid analogous to serum sickness. An Med Interna (Madrid) 1995; 12: 289-90.

7. Ortega Calvo M, Macías Párez V, Merino Kolly MN, Torello Iserte J. Hyperprolactinemia secondary to hypotensive treatment with verapamil. Aten Primaria 2000; 26: 510-1.

8. Maringhini A, Termini A, Patti R, Ciambra M, Biffarella P, Pagliaro L. Enalapril - associated acute pancreatitis: Recurrence after rechallenge. Am J Gastroenterol 1997; 92: 166-7.

9. Carnovale A, Esposito P, Bassano P, Russo L, Uomo G. Enalapril-induced acute recurrent pancreatitis. Dig Liver Dis 2003; 35: 55-7.

10. Segal AW. How neutrophils kill microbes. Annu Rev Immunol 2005; 23: $197-223$.

11. Casimir C, Chetty M, Bohler MC, García R, Fischer A, Griscelli C, et al. Identification of the defective NADPH-oxidase component in chronic granulomatous disease: A study of 57 European families. Eur J Clin Invest 1992; 22: 403-6.

12. Barrot E, Ortega-Calvo M, Borderas F, Sánchez J, Melero-Ruiz J, Sánchez-Sánchez B. Bronchocentric granulomatosis as a first clinical manifestation in an adult patient with p67phox deficiency. Respiration 1999; 66: $547-50$.

13. Noguerol P, Leal M, Sosa R. Fourth case of AIDS in haemophilic children in Seville. Lancet 1984; 2: 986.

14. Pérez R, Martínez RL, Pinero A, Sosa R. Sequential treatment with bolus and continuous infusion of recombinant factor VIIa for hip arthroplasty in a patient with haemophilia A and inhibitor. Haemophilia 2002; 8: $822-5$.

15. Ortega-Calvo M, Sosa-Álamo R, Mayol-Deyá A. Acute subarachnoid hemorrhage associated with platelet storage pool disease and the hemoglobinopathy caused by beta - thalassemia minor. J Stroke Cerebrovasc Dis 2004; 13: 189-91.

16. Ortega Calvo M, Cayuela Domínguez A, Macías Pérez V, González Álvarez M. Congenital myotonic dystrophy in a rural health area. Aten Primaria 2004; 33: 53-4.

17. López de Munain A, Blanco A, Emparanza JI, Poza JJ, Marti Masso JF, Cobo A, et al. Prevalence of myotonic dystrophy in Guipuzcoa (Basque Country, Spain). Neurol 1993; 43: 1573-6.

18. Burcet J, Canellas F, Cavaller G, Vich M.: Epidemiologic study of myotonic dystrophy on the island of Mallorca. Neurologia.1992; 7:61-64

19. Castilla-Guerra L, Fernández-Moreno MC, López-Chozas JM. Treating patients with acute stroke in a rural area. The role played by primary health care. Rev Neurol 2004; 38: 997-8.

20. Olivar Castrillón C, González Morán S, Martínez Suárez MM. The factors related to job satisfaction and professional burnout in the primary care physicians of Asturias. Aten Primaria 1999; 24: 352-9.

21. Wood L, Martínez C. The general practice research database: Role in pharmacovigilance. Drug Saf 2004; 27: 871-81.

22. Aspinall MB, Whittle J, Aspinall SL, Maher RL Jr, Good CB. Improving adverse-drug-reaction reporting in ambulatory care clinics at a Veterans Affairs hospital. Am J Health Syst Pharm 2002; 59: 841-5. 
23. Vargas Castrillon E. Adverse drug effect reporting. An Med Interna (Madrid) 2002; 19: 273-4.

24. Matamoros Flori N, Mila Llambi J, Pons de Ves J. Primary immunodeficiencies in Spain. Data of the Spanish Registry of Primary Immunodeficiencies. REDIP 1980-1999 (Registro Español de Immunodeficiencias Primarias). Med Clin (Barc) 2000; 114: 96-100.

25. Mila Llambi J, Etxagibel Galdos A, Matamoros Flori N. The Spanish Registry of Primary Immunodeficiencies (REDIP). Allergol Immunopathol (Madr) 2001; 29: 122-5.

26. Registro Español de Inmunodeficiencias Primarias. Disponible en: http://web.hsd.es/redip.

27. Winkelstein JA, Marino MC, Johnston RB Jr, Boyle J, Curnutte J, Gallin JI, et al. Chronic granulomatous disease. Report on a national registry of 368 patients. Medicine (Baltimore) 2000; 79: 155-69.

28. Hasui M. Chronic granulomatous disease in Japan: incidence and natural history. The Study Group of Phagocyte Disorders of Japan. Pediatr Int. 1999; 41: 589-93.

29. El Bekay R, Álvarez M, Monteseirin J, Alba G, Chacón P, Vega A, et al. Oxidative stress is a critical mediator of the angiotensin II signal in human neutrophils: Involvement of mitogen-activated protein kinase, calcineurin, and the transcription factor NF-kappaB. Blood 2003; 102: 662-71.

30. Alba G, El Bekay R, Álvarez-Maqueda M, Chacón P, Vega A, Monteseirin J, et al. Stimulators of AMP-activated protein kinase inhibit the respiratory burst in human neutrophils. FEBS Lett 2004; 573: 219-25.

31. Vega A, Chacón P, Monteseirin J, El Bekay R, Álvarez M, Alba G, et al. A new role for monoamine oxidases in the modulation of macrophage-inducible nitric oxide synthase gene expression. J Leukoc Biol 2004; 75: 1093-101.

32. Barroso S, Sánchez B, Álvarez AJ, López-Trascasa M, Lanuza A, Luque R, et al. Complement component C7 deficiency in two Spanish families. Immunology 2004; 113: 518-23.
33. Moltyaner Y, Geerts WH, Chamberlain DW, Heyworth PG, Noack D, Rae J, et al. Underlying chronic granulomatous disease in a patient with bronchocentric granulomatosis. Thorax 2003; 58: 1096-8.

34. van Gijn J, Rinkel GJ. Subarachnoid haemorrhage: Diagnosis, causes and management. Brain 2001; 124: 249-78.

35. Tsementzis SA, Marsh J. Spontaneous subarachnoid haemorrhage occurring in association with a platelet function disorder. Neurosurg Rev 1991; 14: 57-9.

36. Brunner HG, Smeets HJ, Nillesen W, van Oost BA, van den Biezenbos JB, Joosten EM, et al. Myotonic dystrophy. Predictive value of normal results on clinical examination. Brain 1991; 114: 2303-11.

37. Giordano M, Comoli AM, De Angelis MS, Mutani R, Sebastiani F, Richiardi PM. Reassessment of the specificity of lens opacities in myotonic dystrophy. Ophthalmic Res 1996; 28: 224-9.

38. Cobo AM, Poza JJ, Blanco A, López de Munain A, Saenz A, Azpitarte $\mathrm{M}$, et al. Frequency of myotonic dystrophy gene carriers in cataract patients. J Med Genet 1996; 33: 221-3.

39. Siciliano G, Manca M, Gennarelli M, Angelini C, Rocchi A, Iudice A, et al. Epidemiology of myotonic dystrophy in Italy: re-apprisal after genetic diagnosis. Clin Genet 2001; 59: 344-49.

40. Fernández Fernández I. Investigación en atención primaria. Med Clin (Barc) 2005; 124: 57-60.

41. Álvarez Pasquín MJ, Mayer Pujadas MA, Llor Vila C. ¿Qué papel desempeña la atención primaria en el abordaje y el control de nuevas enfermedades? Gripe aviar, síndrome respiratorio agudo grave, bioterrorismo y otras. Aten Primaria 2005; 15; 35: 204-7.

42. Domínguez A. Bioterrorismo, salud pública y vacunas. Vacunas 2002; 3: 45-7.

43. Madoff LC, Woodall JP. The Internet and the Global Monitoring of Emerging Diseases: Lessons from the First 10 Years of ProMED-mail. Arch Med Res 2005; 36: 724-30. 